\title{
BIOATIVIDADE DE PRODUTOS FITOSSANITÁRIOS UTILIZADOS NA CULTURA DO TOMATEIRO (Lycopersicon esculentum MILL.) A Trichogramma pretiosum Riley, 1879 (HYMENOPTERA: TRICHOGRAMMATIDAE) NAS GERAÇÕES F E F $_{2}$
}

\author{
GERALDO ANDRADE CARVALHO \\ JOSÉ ROBERTO POSTALI PARRA ${ }^{2}$ \\ GILBERTO CASADEI BAPTISTA ${ }^{2}$
}

\begin{abstract}
RESUMO - A bioatividade de dezoito produtos químicos utilizados no controle de pragas e doenças do tomateiro, sobre duas linhagens de Trichogramma pretiosum Riley, 1879 ( $\mathrm{L}_{9}=$ Alegre, ES e $\mathrm{L}_{10}=$ Venda Nova do Imigrante, ES), nas gerações $\mathrm{F}_{1}$ e $\mathrm{F}_{2}$, foi investigada em laboratório. Ovos de Anagasta kuehniella (Zeller) contendo o parasitóide em diferentes estágios de desenvolvimento (ovo-larva, pré-pupa e pupa) foram tratados por meio de imersão nas respectivas caldas químicas. Os inseticidas triflumuron, clorfluazuron, deltametrina, Bacillus thuringiensis, lambdacialotrina, teflubenzuron, acefato, pirimicarb e ciromazina, e os fungicidas benomil, iprodiona, clorotalonil e dimetomorf, independente da linhagem, não reduziram a longevidade das fêmeas
\end{abstract}

de $T$. pretiosum da geração $\mathrm{F}_{1}$. Os inseticidas abamectin, cartap, metamidofós e lambdacialotrina afetaram a razão sexual de indivíduos da geração $F_{1}$ e não reduziram a taxa de emergência de parasitóides da $F_{2}$, independente do estágio de desenvolvimento e da origem da população de T. pretiosum. Parasitóides de Venda Nova do Imigrante, $\mathrm{ES}\left(\mathrm{L}_{10}\right)$ mostraram-se mais susceptíveis que os de Alegre, ES ( $\left.\mathrm{L}_{9}\right)$ aos efeitos dos compostos avaliados. De modo geral, a fase de pupa de $T$. pretiosum, independente da população, apresentou maior tolerância aos produtos testados. Recomenda-se a realização de novos testes para outras populações desse parasitóide que serão utilizadas no controle de pragas, pois podem responder de forma diversa aos produtos fitossanitários avaliados.

TERMOS PARA INDEXAÇÃO: Trichogramma, pesticidas, seletividade, tomate.

\section{BIOACTIVITY OF PESTICIDES USED IN TOMATO (Lycopersicon esculentum MILL.) CROP TO Trichogramma pretiosum Riley, 1879 (HYMENOPTERA: TRICHOGRAMMATIDAE) IN F AND F $_{2}$ GENERATIONS}

\begin{abstract}
The bioactivity of eighteen pesticides commonly used to pests and diseases control on tomato, in two strains of Trichogramma pretiosum Riley, 1879 $\left(\mathrm{L}_{9}=\right.$ Alegre, $\mathrm{ES}$ and $\mathrm{L}_{10}=$ Venda Nova do Imigrante, $\mathrm{ES}$, Brazil) in $F_{1}$ and $F_{2}$ generations, was evaluated under laboratory conditions. The bioassays were conducted in a climatic chamber at $25 \pm 2{ }^{\circ} \mathrm{C}, 60 \pm 10 \% \mathrm{RH}$ and 14 hours of photophase. Eggs of Anagasta kuehniella (Zeller) containing the parasitoid in different phases of development (egg-larva, pre-pupa and pupa) were treated by immersion in aqueous dilutions of the products. The insecticides triflumuron, chlorfluazuron, deltamethrin, Bacillus thuringiensis, lambdacyalothrin, abamectin,
\end{abstract}

cartap and methamidophos, did not decrease the longevity of females $T$. pretiosum in $\mathrm{F}_{1}$ generation independently of the parasitoid strain. Abamectin, cartap, methamidophos and lambdacyalothrin affected the sexual ratio in $F_{1}$, and did not decrease the emergence of parasitoids in $F_{2}$ generation, independently of stage of development and origin. The $\mathrm{L}_{10}$ strain was more susceptible to all pesticides as compared to $\mathrm{L}_{9}$. The pupal stage of $T$. pretiosum was more tolerant to the products, independently of its origin. It is recommended new tests with other strains of this parasitoid which may respond differently to the products used, before field recomendations are made.

INDEX TERMS: Trichogramma, pesticides, selectivity, tomato.

1. Professor do Departamento de Entomologia da UNIVERSIDADE FEDERAL DE LAVRAS/UFLA, Caixa Postal 37, 37200-000, Lavras, MG, gacarval@ufla.br.

2. Professor do Departamento de Entomologia, Fitopatologia e Zoologia Agrícola da ESALQ, Caixa Postal 9, 13418900, Piracicaba, SP, jrpparra@carpa.ciagri.usp.br e cbaptis@carpa.ciagri.usp.br 


\section{INTRODUÇÃO}

$\mathrm{Na}$ cultura do tomateiro existem cerca de 200 espécies de artrópodes, e entre os insetos-pragas associados a ela, vários pertencem à Ordem Lepidoptera, merecendo destaque a traça-do-tomateiro Tuta absoluta (Meyrick) (Lepidoptera: Gelechiidae) (Lange \& Bronson, 1981). Esse inseto-praga vem sendo controlado em alguns países biologicamente mediante liberações inundativas de Trichogramma pretiosum Riley, 1879 (Hymenoptera: Trichogrammatidae) (Amaya-Navarro, 1998; Haji, 1992, 1996, 1997; Faria Júnior, 1992).

Para o sucesso na implementação do Manejo Integrado de Pragas (MIP) nessa cultura utilizando-se o Controle Biológico, é necessário que os produtos fitossanitários utilizados sejam seletivos aos parasitóides, e que estudos nessa área sejam incentivados em diferentes condições (Wetzel \& Dickler, 1994). Dessa forma, o uso de produtos seletivos associados a liberações de espécies do gênero Trichogramma permitirá a otimização do controle de lepidópteros-pragas na cultura do tomateiro (Campbell et al., 1991).

Com este trabalho objetivou-se avaliar os efeitos dos principais produtos fitossanitários utilizados na cultura do tomateiro a duas linhagens ou populações de $T$. pretiosum nas gerações $\mathrm{F}_{1}$ e $\mathrm{F}_{2}$.

\section{MATERIAL E MÉTODOS}

O trabalho foi realizado no Laboratório de Biologia dos Insetos do Departamento de Entomologia da Escola Superior de Agricultura "Luiz de Queiroz" (ESALQ) da Universidade de São Paulo (USP), em Piracicaba, SP. Avaliou-se a seletividade dos principais produtos fitossanitários utilizados na cultura do tomateiro a duas populações de T. pretiosum oriundas do Estado do Espírito Santo, sendo uma de Alegre, que recebeu o código $\mathrm{L}_{9}$, e a outra de Venda Nova do Imigrante, com o código $\mathrm{L}_{10}$.

Os parasitóides recém-emergidos foram criados e multiplicados em ovos de Anagasta kuehniella (Zeller). Os produtos avaliados com as suas concentrações expressas em g i.a./L de água foram: triflumuron $(0,15)$, clorfluazuron $(0,05)$, benomil $(0,5)$, clorotalonil $(1,5)$, deltametrina $(0,0125)$, Bacillus thuringiensis $(0,32)$, mancozeb $(2,4)$, dimetomorf $(0,75)$, lambdacialotrina $(0,025)$, tebufenozide $(0,12)$, teflubenzuron $(0,0375)$, acefato $(0,75)$, pirimicarb $(0,25)$, iprodiona $(0,75)$, metamidofós $(0,6)$, cartap $(0,6)$, ciromazina $(0,1125)$ e abamectin $(0,018)$, empregando-se a maior dosagem recomendada pelo fabricante para o controle de pragas ou doenças da cultura do tomateiro, e no tratamentotestemunha, utilizou-se somente água destilada.

\section{Bioatividade dos produtos fitossanitários so- bre $T$. pretiosum nas gerações $F_{1}$ e $F_{2}$}

As duas populações de $T$. pretiosum foram criadas separadamente, em cubas de vidro de 16 × 12 × 30 $\mathrm{cm}$, para avaliação do efeito dos compostos sobre a fase imatura. Ofereceram-se às fêmeas do parasitóide cerca de 250 ovos de A. kuehniella/cartela de cartolina azul durante 24 horas, na proporção de 1 fêmea do parasitóide: 10 ovos do hospedeiro. Em seguida, as cartelas contendo os ovos parasitados foram retiradas e colocadas em novas cubas, acondicionando-as em câmara climática a $25 \pm 2^{\circ} \mathrm{C}$, UR de $60 \pm 10 \%$ e fotofase de 14 horas.

Dessa forma, ovos de A. kuehniella contendo o parasitóide em diferentes estágios de desenvolvimento (ovo-larva, pré-pupa e pupa) foram submetidos aos diferentes tratamentos. Em cada bioensaio, utilizaram-se 20 repetições por tratamento, com parcelas constituídas de uma cartela contendo cerca de 250 ovos supostamente parasitados.

Os ovos contendo os parasitóides foram imersos nas caldas químicas por um período de cinco segundos. Em seguida, as cartelas foram mantidas em condições ambientais por cerca de uma hora para eliminação do excesso de umidade da superfície dos ovos. Posteriormente, elas foram individualizadas em tubos de vidro $(8,5 \mathrm{~cm} \times 2,5 \mathrm{~cm})$, que foram dispostos em câmaras climatizadas.

Após a emergência dos indivíduos da geração $F_{1}$, foram individualizadas 20 fêmeas em tubos de vidro $(8,5 \mathrm{~cm} \times 2,5 \mathrm{~cm})$, por tratamento, que foram alimentadas com mel adicionado no interior dos tubos. Para cada fêmea foram oferecidos cerca de 125 ovos de $\mathrm{A}$. $\mathrm{ku}$ ehniella não-tratados com inseticidas ou fungicidas, distribuídos em cartelas de cartolina azul, por um período de 24 horas. Após esse período, as cartelas foram transferidas para novos tubos. Avaliaram-se a razão sexual dos parasitóides da geração $F_{1}$, a longevidade das fêmeas dessa geração que realizaram o parasitismo e também a porcentagem de emergência dos indivíduos da geração $\mathrm{F}_{2}$.

As análises de variância foram efetuadas em computador pelo programa SANEST. Os dados foram analisados, testando-se a normalidade dos erros pelo teste de Lilliefors e homogeneidade das variâncias pelo teste de Bartlet, sendo as médias comparadas pelo teste de Tukey ao nível de $5 \%$ de probabilidade. Os dados de 
contagem foram transformados para $\sqrt{x}$ ou $\sqrt{x+0,5}$ e os de porcentagem para arco-seno $\sqrt{x / 100}$.

\section{RESULTADOS E DISCUSSÃO}

\section{Bioatividade de produtos fitossanitários sobre a razão sexual de $T$. pretiosum $\left(F_{1}\right)$, tratados nos estágios de ovo-larva, pré-pupa e pupa, em ovos de A. kuehniella}

Observou-se que, independentemente do estágio de desenvolvimento do parasitóide, todos os produtos fitossanitários apresentados na Tabela 1 não afetaram a razão sexual de T. pretiosum $\left(\mathrm{L}_{9}\right)$. Resultados semelhantes também foram obtidos por Parra (1994), que aplicando os inseticidas tebufenozide, teflubenzuron, lambdacialotrina e abamectin sobre os estágios de ovo-larva, pré-pupa e pupa de $T$. pretiosum, não encontrou efeito negativo sobre a razão sexual dos parasitóides emergidos.

Independentemente das épocas de aplicação dos pesticidas, com exceção dos inseticidas metamidofós, cartap e lambdacialotrina, os demais produtos não afetaram a razão sexual dos indivíduos da $\mathrm{L}_{10}$ (Tabela 2). O metamidofós, quando aplicado sobre ovos de $A$. kuehniella contendo os parasitóides no estágio de ovolarva, reduziu o número de fêmeas da população em comparação com o tratamento-testemunha. O mesmo ocorreu para o inseticida cartap quando aplicado sobre as fases de ovo-larva e pré-pupa de $T$. pretiosum e também com a lambdaciolotrina sobre o parasitóide na fase de pré-pupa. A razão sexual média apresentada por esses compostos foi de $0,35,0,36$ e 0,35 , contra $0,42,0,36$ e 0,41 do tratamento-testemunha, respectivamente.

\section{Bioatividade de produtos fitossanitários na longevidade de $T$. pretiosum $\left(\mathrm{F}_{1}\right)$ tratado nos estágios de ovo-larva, pré-pupa e pupa, em ovos de A. kuehniella}

A longevidade de fêmeas de $T$. pretiosum $\left(\mathrm{L}_{9}\right)$, provenientes do tratamento dos ovos do hospedeiro alternativo entre 0-24 horas após parasitismo, foi afetada pelos inseticidas abamectin e metamidofós, e pelo fungicida mancozeb, com reduções médias de 84,80\%, $37,51 \%$ e $24,22 \%$, respectivamente (Tabela 3). Esse fungicida também foi prejudicial às fêmeas do parasitóide, quando aplicado entre 72-96 horas depois do parasitismo, reduzindo a longevidade do parasitóide em cerca de 21,19\%, ao contrário dos dois inseticidas. O inseticida tebufenozide, quando aplicado sobre esse estágio, reduziu a longevidade das fêmeas em torno de $21,34 \%$. Já aquelas provenientes do tratamento sobre pupas foram tolerantes a todos os produtos fitossanitários utilizados, apresentando longevidades médias ao redor de 11 dias (Tabela 3). A seletividade dos pesticidas sobre a longevidade de fêmeas do $T$. pretiosum da $\mathrm{L}_{9}$, tratadas no estágio de pupa, deve ser levada em consideração na escolha da melhor época de aplicação dos defensivos agrícolas. No caso da utilização do Controle Biológico Aplicado com essa linhagem de parasitóide e, se for necessária a aplicação dos produtos fitossanitários avaliados, deve-se utilizá-los de preferência um ou dois dias antes da emergência dos parasitóides (estágio de pupa).

Os inseticidas abamectin e cartap, quando aplicados entre 0-24 horas após o parasitismo de T. pretiosum, da $\mathrm{L}_{10}$, foram tóxicos às fêmeas, causando reduções na longevidade de $61,68 \%$ e $25,33 \%$, respectivamente. A longevidade de fêmeas provenientes do tratamento sobre o estágio de pré-pupa foi afetada pelos inseticidas abamectin, metamidofós, cartap e tebufenozide. Aquelas provenientes do tratamento entre 168-192 horas após parasitismo, tiveram a longevidade afetada somente pelos inseticidas abamectin, cartap e tebufenozide, com reduções médias de $54,48 \%, 27,22 \%$ e $84,51 \%$, respectivamente (Tabela 4).

De modo geral, ocorreram diferenças significativas entre as duas linhagens aos produtos fitossanitários, e entre os próprios estágios de cada uma delas, sendo o de pupa da $\mathrm{L}_{9}$ o mais tolerante a todos os pesticidas estudados (Tabela 3). Para a $\mathrm{L}_{10}$ de $T$. pretiosum, os inseticidas abamectin e cartap, independente da época de aplicação (ovo-larva, pré-pupa e pupa), afetaram a longevidade das fêmeas (Tabela 4).

Com relação aos demais compostos avaliados, não se observou efeito adverso sobre esse parâmetro biológico. Assim, os inseticidas reguladores de crescimento de insetos não afetaram a longevidade das fêmeas das duas linhagens, concordando com observações de Carvalho et al. (1994) com triflumuron, clorfluazuron e ciromazina, diferindo dos resultados obtidos por Zaki \& Gesraha (1987), os quais verificaram para diflubenzuron, sobre Trichogramma evanescens Westwood, 1833 redução de aproximadamente $44,00 \%$. Esses autores utilizaram dosagens acima das utilizadas no presente trabalho, o que pode ter contribuído para a discrepância de resultados, além das espécies serem diferentes.

De maneira geral, os parasitóides da $\mathrm{L}_{10}$ mostraram-se mais susceptíveis ao efeito dos compostos avaliados. 
TABELA 1 - Razão sexual média de Trichogramma pretiosum, da $\mathrm{L}_{9}$, geração $\mathrm{F}_{1}$, tratados nos estágios de ovolarva, pré-pupa e pupa, em ovos de Anagasta kuehniella.

\begin{tabular}{|c|c|c|c|c|}
\hline \multirow{3}{*}{$\begin{array}{c}\text { Produto } \\
\text { fitossanitário }\end{array}$} & \multicolumn{3}{|c|}{ Período de desenvolvimento do parasitóide } & \multirow{3}{*}{ Média } \\
\hline & $\begin{array}{c}\text { 0-24 h } \\
\text { (ovo-larva) }\end{array}$ & $\begin{array}{c}\text { 72-96 h } \\
\text { (pré-pupa) }\end{array}$ & $\begin{array}{l}\text { 168-192 h } \\
\text { (pupa) }\end{array}$ & \\
\hline & \multicolumn{3}{|c|}{ Razão sexual* } & \\
\hline testemunha & $0,61 \pm 0,03 \mathrm{ab}$ & $0,71 \pm 0,03 \mathrm{a}$ & $0,61 \pm 0,02 \mathrm{a}$ & 0,64 \\
\hline clorfluazuron & $0,62 \pm 0,05 \mathrm{ab}$ & $0,58 \pm 0,02 \mathrm{ab}$ & $0,58 \pm 0,02 \mathrm{ab}$ & 0,59 \\
\hline teflubenzuron & $0,72 \pm 0,03 \mathrm{a}$ & $0,65 \pm 0,04 \mathrm{ab}$ & $0,57 \pm 0,04 \mathrm{ab}$ & 0,65 \\
\hline triflumuron & $0,59 \pm 0,03 \mathrm{ab}$ & $0,57 \pm 0,03 \mathrm{ab}$ & $0,58 \pm 0,03 \mathrm{ab}$ & 0,58 \\
\hline B. thuringiensis & $0,64 \pm 0,03 \mathrm{ab}$ & $0,62 \pm 0,02 \mathrm{ab}$ & $0,60 \pm 0,02 \mathrm{a}$ & 0,62 \\
\hline ciromazina & $0,62 \pm 0,02 \mathrm{ab}$ & $0,56 \pm 0,04 \mathrm{ab}$ & $0,56 \pm 0,02 \mathrm{ab}$ & 0,58 \\
\hline deltametrina & $0,71 \pm 0,03 \mathrm{a}$ & $0,67 \pm 0,03 \mathrm{ab}$ & $0,53 \pm 0,05 \mathrm{ab}$ & 0,64 \\
\hline Acefato & $0,73 \pm 0,03 \mathrm{a}$ & $0,62 \pm 0,02 \mathrm{ab}$ & $0,59 \pm 0,02 \mathrm{a}$ & 0,65 \\
\hline abamectin & $0,62 \pm 0,09 \mathrm{ab}$ & $0,66 \pm 0,04 \mathrm{ab}$ & $0,65 \pm 0,03 \mathrm{a}$ & 0,65 \\
\hline metamidofós & $0,61 \pm 0,02 \mathrm{ab}$ & $0,72 \pm 0,03 \mathrm{a}$ & $0,60 \pm 0,02 \mathrm{a}$ & 0,65 \\
\hline C.V. $(\%)$ & 23,29 & 21,54 & 20,75 & \\
\hline testemunha & $0,47 \pm 0,01 \mathrm{~b}$ & $0,370 \pm 0,02 \mathrm{ab}$ & $0,38 \pm 0,01 \mathrm{a}$ & 0,38 \\
\hline Cartap & $0,49 \pm 0,03 \mathrm{a}$ & $0,41 \pm 0,03$ a & $0,38 \pm 0,03 \mathrm{a}$ & 0,43 \\
\hline Benomil & $0,40 \pm 0,01 \mathrm{ab}$ & $0,36 \pm 0,02 \mathrm{ab}$ & $0,36 \pm 0,01 \mathrm{ab}$ & 0,37 \\
\hline clorotalonil & $0,40 \pm 0,01 \mathrm{ab}$ & $0,38 \pm 0,02 \mathrm{ab}$ & $0,36 \pm 0,02 \mathrm{ab}$ & 0,38 \\
\hline mancozeb & $0,38 \pm 0,01 b$ & $0,39 \pm 0,01 \mathrm{a}$ & $0,39 \pm 0,01 \mathrm{a}$ & 0,39 \\
\hline Iprodiona & $0,45 \pm 0,02 \mathrm{ab}$ & $0,37 \pm 0,02 \mathrm{ab}$ & $0,42 \pm 0,02 \mathrm{a}$ & 0,41 \\
\hline dimetormorf & $0,40 \pm 0,01 \mathrm{ab}$ & $0,38 \pm 0,02 \mathrm{ab}$ & $0,37 \pm 0,02 \mathrm{ab}$ & 0,38 \\
\hline tebufenozide & $0,41 \pm 0,01 \mathrm{ab}$ & $0,41 \pm 0,02 \mathrm{a}$ & $0,44 \pm 0,01 \mathrm{a}$ & 0,42 \\
\hline lambdacialotrina & $0,37 \pm 0,01 b$ & $0,39 \pm 0,01 \mathrm{a}$ & $0,36 \pm 0,01 \mathrm{ab}$ & 0,37 \\
\hline pirimicarb & $0,39 \pm 0,02 b$ & $0,37 \pm 0,01 \mathrm{ab}$ & $0,38 \pm 0,01 \mathrm{ab}$ & 0,38 \\
\hline C.V. $(\%)$ & 14,86 & 10,46 & 9,11 & \\
\hline
\end{tabular}

Médias seguidas da mesma letra, na coluna, não diferem significativamente entre si pelo teste de Tukey ao nível de $5 \%$ de probabilidade.

*Razão sexual média \pm erro-padrão da média. 
TABELA 2 - Razão sexual média de Trichogramma pretiosum, da $\mathrm{L}_{10}$, geração $\mathrm{F}_{1}$, tratados nos estágios de ovolarva, pré-pupa e pupa, em ovos de Anagasta kuehniella.

\begin{tabular}{|c|c|c|c|c|}
\hline \multirow{3}{*}{$\begin{array}{c}\text { Produto } \\
\text { fitossanitário }\end{array}$} & \multicolumn{3}{|c|}{ Período de desenvolvimento do parasitóide } & \multirow{3}{*}{ Média } \\
\hline & $\begin{array}{c}\text { 0-24 h } \\
\text { (ovo-larva) }\end{array}$ & $\begin{array}{c}\text { 72-96 h } \\
\text { (pré-pupa) }\end{array}$ & $\begin{array}{c}\text { 168-192 h } \\
\text { (pupa) }\end{array}$ & \\
\hline & \multicolumn{3}{|c|}{ Razão sexual* } & \\
\hline testemunha & $0,40 \pm 0,01 \mathrm{a}$ & $0,41 \pm 0,02 \mathrm{a}$ & $0,45 \pm 0,01 \mathrm{a}$ & 0,42 \\
\hline clorfluazuron & $0,34 \pm 0,01 \mathrm{ab}$ & $0,45 \pm 0,02 \mathrm{a}$ & $0,46 \pm 0,02 \mathrm{a}$ & 0,41 \\
\hline teflubenzuron & $0,34 \pm 0,02 \mathrm{ab}$ & $0,47 \pm 0,02 \mathrm{a}$ & $0,47 \pm 0,02$ a & 0,43 \\
\hline triflumuron & $0,38 \pm 0,02 \mathrm{ab}$ & $0,33 \pm 0,03 \mathrm{ab}$ & $0,42 \pm 0,02 \mathrm{a}$ & 0,37 \\
\hline B. thuringiensis & $0,37 \pm 0,04 \mathrm{ab}$ & $0,45 \pm 0,01 \mathrm{a}$ & $0,44 \pm 0,02 \mathrm{a}$ & 0,41 \\
\hline ciromazina & $0,36 \pm 0,01 \mathrm{ab}$ & $0,44 \pm 0,03$ a & $0,44 \pm 0,03$ a & 0,42 \\
\hline deltametrina & $0,34 \pm 0,02 \mathrm{ab}$ & $0,39 \pm 0,03 \mathrm{ab}$ & $0,40 \pm 0,02 \mathrm{a}$ & 0,38 \\
\hline Acefato & $0,34 \pm 0,03 \mathrm{ab}$ & $0,39 \pm 0,02 \mathrm{ab}$ & $0,45 \pm 0,02 \mathrm{a}$ & 0,39 \\
\hline abamectin & $0,34 \pm 0,05 \mathrm{ab}$ & $0,49 \pm 0,03$ a & $0,48 \pm 0,02 \mathrm{a}$ & 0,44 \\
\hline metamidofós & $0,27 \pm 0,04 \mathrm{~b}$ & $0,36 \pm 0,03 \mathrm{ab}$ & $0,41 \pm 0,02 \mathrm{a}$ & 0,35 \\
\hline C.V. (\%) & 20,61 & 18,57 & 16,12 & \\
\hline testemunha & $0,41 \pm 0,01 \mathrm{a}$ & $0,46 \pm 0,03$ a & $0,36 \pm 0,01 \mathrm{a}$ & 0,41 \\
\hline Cartap & $0,33 \pm 0,02 \mathrm{~b}$ & $0,34 \pm 0,02 \mathrm{~b}$ & $0,41 \pm 0,01 \mathrm{a}$ & 0,36 \\
\hline Benomil & $0,44 \pm 0,01 \mathrm{a}$ & $0,38 \pm 0,01 \mathrm{ab}$ & $0,38 \pm 0,01 \mathrm{a}$ & 0,40 \\
\hline clorotalonil & $0,43 \pm 0,01 \mathrm{a}$ & $0,38 \pm 0,01 \mathrm{ab}$ & $0,40 \pm 0,01 \mathrm{a}$ & 0,41 \\
\hline mancozeb & $0,37 \pm 0,02 \mathrm{ab}$ & $0,38 \pm 0,01 \mathrm{ab}$ & $0,39 \pm 0,02 \mathrm{a}$ & 0,38 \\
\hline iprodiona & $0,44 \pm 0,03 \mathrm{a}$ & $0,42 \pm 0,02 \mathrm{ab}$ & $0,44 \pm 0,01 \mathrm{a}$ & 0,44 \\
\hline dimetormorf & $0,38 \pm 0,01 \mathrm{ab}$ & $0,36 \pm 0,01 \mathrm{ab}$ & $0,34 \pm 0,02 \mathrm{a}$ & 0,36 \\
\hline tebufenozide & $0,41 \pm 0,01 \mathrm{a}$ & $0,38 \pm 0,01 \mathrm{ab}$ & $0,39 \pm 0,01 \mathrm{a}$ & 0,39 \\
\hline lambdacialotrina & $0,41 \pm 0,05 \mathrm{a}$ & $0,18 \pm 0,04 \mathrm{c}$ & $0,46 \pm 0,05 \mathrm{a}$ & 0,35 \\
\hline pirimicarb & $0,41 \pm 0,01 \mathrm{a}$ & $0,37 \pm 0,01 \mathrm{ab}$ & $0,40 \pm 0,01 \mathrm{a}$ & 0,39 \\
\hline C.V. (\%) & 19,24 & 23,91 & 19,89 & \\
\hline
\end{tabular}

Médias seguidas da mesma letra, na coluna, não diferem significativamente entre si pelo teste de Tukey ao nível de $5 \%$ de probabilidade.

*Razão sexual média \pm erro-padrão da média. 
TABELA 3 - Longevidade média de fêmeas de Trichogramma pretiosum, da $\mathrm{L}_{9}$, geração $\mathrm{F}_{1}$, tratadas nos estágios de ovo-larva, pré-pupa e pupa, em ovos de Anagasta kuehniella.

\begin{tabular}{|c|c|c|c|c|}
\hline \multirow{3}{*}{$\begin{array}{c}\text { Produto } \\
\text { fitossanitário }\end{array}$} & \multicolumn{3}{|c|}{ Período de desenvolvimento do parasitóide } & \multirow{3}{*}{ Média } \\
\hline & $\begin{array}{c}\text { 0-24 h } \\
\text { (ovo-larva) }\end{array}$ & $\begin{array}{c}\text { 72-96 h } \\
\text { (pré-pupa) }\end{array}$ & $\begin{array}{c}\text { 168-192 h } \\
\text { (pupa) }\end{array}$ & \\
\hline & \multicolumn{3}{|c|}{ Longevidade (dias)* } & \\
\hline testemunha & $11,25 \pm 0,62 \mathrm{a}$ & $10,96 \pm 1,27 \mathrm{ab}$ & $10,21 \pm 1,52 \mathrm{ab}$ & 10,71 \\
\hline clorfluazuron & $9,24 \pm 0,65 \mathrm{ab}$ & $13,49 \pm 0,91 \mathrm{ab}$ & $11,25 \pm 1,37 \mathrm{ab}$ & 11,26 \\
\hline teflubenzuron & $11,01 \pm 0,63$ a & $11,80 \pm 1,11 \mathrm{ab}$ & $13,25 \pm 0,89 \mathrm{a}$ & 12,00 \\
\hline triflumuron & $9,73 \pm 0,72 \mathrm{ab}$ & $14,52 \pm 0,68$ a & $11,50 \pm 1,00 \mathrm{ab}$ & 11,83 \\
\hline B. thuringiensis & $10,75 \pm 0,83 \mathrm{ab}$ & $14,13 \pm 0,64 \mathrm{ab}$ & $12,63 \pm 0,68 \mathrm{a}$ & 12,47 \\
\hline ciromazina & $9,96 \pm 0,55 \mathrm{ab}$ & $11,39 \pm 1,15 \mathrm{ab}$ & $14,20 \pm 0,65 \mathrm{a}$ & 11,79 \\
\hline deltametrina & $10,16 \pm 0,70 \mathrm{ab}$ & $15,44 \pm 0,60$ a & $13,72 \pm 0,71 \mathrm{a}$ & 13,02 \\
\hline Acefato & $8,28 \pm 0,96 \mathrm{ab}$ & $14,43 \pm 0,58 \mathrm{ab}$ & $11,66 \pm 0,62 \mathrm{ab}$ & 11,32 \\
\hline abamectin & $1,71 \pm 0,21 \mathrm{c}$ & $6,09 \pm 1,22 \mathrm{~b}$ & $5,49 \pm 0,67 \mathrm{~b}$ & 4,47 \\
\hline metamidofós & $7,03 \pm 0,88 \mathrm{~b}$ & $11,96 \pm 1,22 \mathrm{ab}$ & $8,04 \pm 1,26 \mathrm{ab}$ & 8,90 \\
\hline C.V. $(\%)$ & 19,90 & 14,96 & 12,89 & \\
\hline testemunha & $11,48 \pm 0,19 \mathrm{a}$ & $13,21 \pm 0,33 \mathrm{a}$ & $11,26 \pm 0,51 \mathrm{a}$ & 11,97 \\
\hline Cartap & $10,82 \pm 0,24 \mathrm{ab}$ & $12,78 \pm 0,43 \mathrm{ab}$ & $12,35 \pm 0,34 \mathrm{a}$ & 11,97 \\
\hline Benomil & $10,26 \pm 0,26 \mathrm{ab}$ & $11,52 \pm 0,73 \mathrm{ab}$ & $11,10 \pm 0,43 \mathrm{a}$ & 10,95 \\
\hline clorotalonil & $10,39 \pm 0,34 \mathrm{ab}$ & $10,67 \pm 0,91 \mathrm{ab}$ & $10,42 \pm 0,68 \mathrm{a}$ & 10,49 \\
\hline mancozeb & $8,70 \pm 0,45 \mathrm{~b}$ & $10,41 \pm 0,78 \mathrm{~b}$ & $11,54 \pm 0,44 \mathrm{a}$ & 10,22 \\
\hline Iprodiona & $9,90 \pm 0,43 \mathrm{ab}$ & $10,72 \pm 0,93 \mathrm{ab}$ & $10,56 \pm 0,77 \mathrm{a}$ & 10,39 \\
\hline dimetormorf & $11,51 \pm 0,29 \mathrm{a}$ & $11,79 \pm 0,79 \mathrm{ab}$ & $11,87 \pm 0,40 \mathrm{a}$ & 11,72 \\
\hline tebufenozide & $9,03 \pm 0,33 \mathrm{ab}$ & $10,46 \pm 0,99 \mathrm{~b}$ & $12,65 \pm 0,36 \mathrm{a}$ & 10,66 \\
\hline lambdacialotrina & $11,09 \pm 0,36 \mathrm{ab}$ & $12,74 \pm 0,74 \mathrm{ab}$ & $10,89 \pm 0,51 \mathrm{a}$ & 11,56 \\
\hline pirimicarb & $10,15 \pm 0,31 \mathrm{ab}$ & $12,42 \pm 0,45 \mathrm{ab}$ & $10,58 \pm 0,46 \mathrm{a}$ & 11,03 \\
\hline C.V. (\%) & 12,54 & 10,21 & 9,98 & \\
\hline
\end{tabular}

Médias seguidas da mesma letra, na coluna, não diferem significativamente entre si pelo teste de Tukey ao nível de $5 \%$ de probabilidade.

*Longevidade média \pm erro-padrão da média. 
TABELA 4 - Longevidade média de fêmeas de Trichogramma pretiosum, da $\mathrm{L}_{10}$, geração $\mathrm{F}_{1}$, tratadas nos estágios de ovo-larva, pré-pupa e pupa, em ovos de Anagasta kuehniella.

\begin{tabular}{|c|c|c|c|c|}
\hline \multirow{3}{*}{$\begin{array}{c}\text { Produto } \\
\text { Fitossanitário }\end{array}$} & \multicolumn{3}{|c|}{ Período de desenvolvimento do parasitóide } & \multirow{3}{*}{ Média } \\
\hline & $\begin{array}{c}\text { 0-24 h } \\
\text { (ovo-larva) }\end{array}$ & $\begin{array}{c}72-96 \text { h } \\
\text { (pré-pupa) }\end{array}$ & $\begin{array}{c}\text { 168-192 h } \\
\text { (pupa) }\end{array}$ & \\
\hline & \multicolumn{3}{|c|}{ Longevidade (dias)* } & \\
\hline testemunha & $8,30 \pm 0,72 \mathrm{a}$ & $11,40 \pm 0,64 a b$ & $7,14 \pm 0,79 a$ & 8,86 \\
\hline clorfluazuron & $9,36 \pm 0,59 \mathrm{a}$ & $8,27 \pm 1,29 \mathrm{bcd}$ & $9,74 \pm 0,47 a$ & 9,11 \\
\hline teflubenzuron & $10,35 \pm 0,45$ a & $9,67 \pm 1,09 \mathrm{abc}$ & $9,00 \pm 0,52 \mathrm{a}$ & 9,67 \\
\hline triflumuron & $8,88 \pm 0,70$ a & $12,18 \pm 0,63 \mathrm{ab}$ & $8,52 \pm 0,36 a$ & 9,79 \\
\hline B. thuringiensis & $9,57 \pm 0,60 \mathrm{a}$ & $12,84 \pm 0,39 \mathrm{a}$ & $8,18 \pm 0,59 a$ & 10,11 \\
\hline ciromazina & $8,49 \pm 0,91 \mathrm{a}$ & $9,76 \pm 0,87 \mathrm{abc}$ & $8,63 \pm 0,50 \mathrm{a}$ & 8,95 \\
\hline deltametrina & $9,56 \pm 0,79 \mathrm{a}$ & $11,19 \pm 0,66 \mathrm{ab}$ & $6,37 \pm 0,81 \mathrm{a}$ & 8,93 \\
\hline Acefato & $7,94 \pm 0,78 \mathrm{a}$ & $11,56 \pm 0,59 \mathrm{ab}$ & $7,93 \pm 0,51 \mathrm{a}$ & 9,07 \\
\hline abamectin & $3,18 \pm 0,46 \mathrm{~b}$ & $5,58 \pm 1,14 \mathrm{~d}$ & $3,25 \pm 0,66 b$ & 4,00 \\
\hline metamidofós & $8,36 \pm 0,86$ a & $6,28 \pm 1,16 \mathrm{~cd}$ & $6,37 \pm 0,83 a$ & 6,97 \\
\hline C.V. $(\%)$ & 21,73 & 18,91 & 17,54 & \\
\hline testemunha & $9,75 \pm 0,51 \mathrm{a}$ & $9,95 \pm 0,33 \mathrm{a}$ & $9,81 \pm 0,70 \mathrm{a}$ & 9,83 \\
\hline Cartap & $7,28 \pm 0,75 \mathrm{~b}$ & $7,39 \pm 0,24 \mathrm{~b}$ & $7,14 \pm 0,30 \mathrm{~b}$ & 6,27 \\
\hline Benomil & $9,46 \pm 0,27 \mathrm{a}$ & $9,34 \pm 0,47 \mathrm{ab}$ & $9,46 \pm 0,29 \mathrm{a}$ & 9,42 \\
\hline clorotalonil & $9,45 \pm 0,49 \mathrm{a}$ & $8,01 \pm 0,23 \mathrm{ab}$ & $8,66 \pm 0,39 a b$ & 8,70 \\
\hline mancozeb & $9,87 \pm 0,52 \mathrm{a}$ & $8,16 \pm 0,24 \mathrm{ab}$ & $8,74 \pm 0,48 a b$ & 8,91 \\
\hline iprodiona & $8,17 \pm 0,54 \mathrm{ab}$ & $8,20 \pm 0,29 \mathrm{ab}$ & $9,89 \pm 0,45 \mathrm{a}$ & 8,74 \\
\hline dimetormorf & $9,04 \pm 0,53 \mathrm{ab}$ & $8,84 \pm 0,45 \mathrm{ab}$ & $9,06 \pm 0,59 a b$ & 8,98 \\
\hline tebufenozide & $8,22 \pm 0,56 \mathrm{ab}$ & $0,71 \pm 0,00 \mathrm{c}$ & $1,52 \pm 0,00 \mathrm{c}$ & 3,48 \\
\hline lambdacialotrina & $9,69 \pm 0,45 \mathrm{a}$ & $7,93 \pm 0,43 \mathrm{ab}$ & $9,85 \pm 0,31 \mathrm{a}$ & 9,14 \\
\hline pirimicarb & $8,43 \pm 0,64 \mathrm{ab}$ & $8,03 \pm 0,44 \mathrm{ab}$ & $8,75 \pm 0,28 \mathrm{ab}$ & 8,30 \\
\hline C.V. $(\%)$ & 12,84 & 17,45 & 16,88 & \\
\hline
\end{tabular}

Médias seguidas da mesma letra não diferem significativamente entre si pelo teste de Tukey ao nível de $5 \%$ de probabilidade.

*Longevidade média \pm erro-padrão da média.

Bioatividade de produtos fitossanitários sobre a emergência de $T$. pretiosum $\left(F_{2}\right)$, provenientes de $F_{1}$ tratadas nos estágios de ovo-larva, pré-pupa e pupa, em ovos de $A$. kuehniella

Os produtos fitossanitários avaliados não afetaram a porcentagem de emergência de $T$. pretiosum, independente da linhagem e da época de aplicação, pois foram observadas emergências que variaram de 91,97\% a 99,13\% (Tabelas 5 e 6). Isso significa que os efeitos da aplicação dos pesticidas sobre o período de ovo-larva e os estágios de pré-pupa e pupa de fêmeas de T. pretiosum da $\mathrm{F}_{1}$, das $\mathrm{L}_{9}$ e $\mathrm{L}_{10}$ provavelmente não foram transmitidos para seus descendentes. Dessa forma, os indivíduos da $F_{1}$ que sobreviveram ao contato com os pesticidas conseguiram se multiplicar normalmente, apresentando altas taxas de parasitismo e emergência de parasitóides da $\mathrm{F}_{2}$.

Em levantamentos bibliográficos realizados, não foi constatado nenhum trabalho que tenha avaliado o efeito de produtos fitossanitários sobre a geração $\mathrm{F}_{2}$ de Trichogramma. Dessa forma, os resultados encontrados no presente trabalho são inéditos e espera-se que os mesmos possam contribuir na seleção de compostos utilizados na cultura do tomateiro e que sejam compatíveis ao controle biológico com $T$. pretiosum. 
TABELA 5 - Porcentagem média de emergência $( \pm \mathrm{EP})$ de Trichogramma pretiosum $\left(\mathrm{F}_{2}\right)$, da $\mathrm{L}_{9}$, provenientes de $\mathrm{F}_{1}$ tratadas nos estágios de ovo-larva, pré-pupa e pupa, em ovos de Anagasta kuehniella.

\begin{tabular}{|c|c|c|c|c|}
\hline \multirow{3}{*}{$\begin{array}{c}\text { Produto } \\
\text { fitossanitário }\end{array}$} & \multicolumn{3}{|c|}{ Período de desenvolvimento do parasitóide } & \multirow{3}{*}{ Média } \\
\hline & $\begin{array}{l}\text { 0-24 h } \\
\text { vo-larva) }\end{array}$ & $\begin{array}{l}72-96 \text { h } \\
\text { pupa) }\end{array}$ & $\begin{array}{c}\text { 168-192 h } \\
\text { pa) }\end{array}$ & \\
\hline & \multicolumn{3}{|c|}{ Porcentagem de emergência } & \\
\hline testemunha & $95,89 \pm 0,87 \mathrm{ab}$ & $96,17 \pm 1,06 \mathrm{ab}$ & $92,67 \pm 5,11 b$ & 95,02 \\
\hline clorfluazuron & $96,67 \pm 0,72 \mathrm{a}$ & $96,404 \pm 2,21 \mathrm{ab}$ & $96,28 \pm 0,70 \mathrm{ab}$ & 96,46 \\
\hline teflubenzuron & $96,57 \pm 0,48 \mathrm{ab}$ & $95,68 \pm 1,79 b$ & $97,46 \pm 0,60 \mathrm{a}$ & 96,61 \\
\hline triflumuron & $96,45 \pm 0,66 \mathrm{ab}$ & $95,40 \pm 0,58 b$ & $96,48 \pm 0,73 \mathrm{ab}$ & 96,13 \\
\hline B. thuringiensis & $93,95 \pm 1,09 \mathrm{ab}$ & $95,00 \pm 1,38 b$ & $95,85 \pm 0,52 \mathrm{ab}$ & 94,96 \\
\hline ciromazina & $94,72 \pm 0,79 \mathrm{ab}$ & $97,11 \pm 0,97 \mathrm{ab}$ & $96,56 \pm 0,40 \mathrm{ab}$ & 96,19 \\
\hline deltametrina & $95,07 \pm 1,93 \mathrm{ab}$ & $96,85 \pm 0,88 \mathrm{ab}$ & $97,77 \pm 0,80 \mathrm{a}$ & 96,65 \\
\hline acefato & $92,24 \pm 0,68 \mathrm{ab}$ & $96,94 \pm 0,64 \mathrm{ab}$ & $97,49 \pm 0,40 \mathrm{a}$ & 96,79 \\
\hline metamidofós & $91,56 \pm 1,58 \mathrm{~b}$ & $99,05 \pm 0,97 \mathrm{a}$ & $96,93 \pm 0,52 \mathrm{ab}$ & 96,45 \\
\hline C.V. $(\%)$ & 8,52 & 7,21 & 7,95 & \\
\hline testemunha & $96,15 \pm 0,86$ abcd & $99,08 \pm 0,43 \mathrm{a}$ & $99,57 \pm 0,36 \mathrm{ab}$ & 98,58 \\
\hline cartap & $98,89 \pm 0,36 \mathrm{a}$ & $98,52 \pm 0,40 a$ & $99,26 \pm 0,46 \mathrm{ab}$ & 98,91 \\
\hline benomil & $98,03 \pm 0,87 \mathrm{ab}$ & $98,03 \pm 0,76 \mathrm{a}$ & $99,02 \pm 0,31 \mathrm{abc}$ & 98,40 \\
\hline clorotalonil & $93,43 \pm 2,21 \mathrm{~cd}$ & $97,33 \pm 2,42 \mathrm{a}$ & $97,85 \pm 0,80 \mathrm{bc}$ & 96,43 \\
\hline mancozeb & $95,73 \pm 0,67 \mathrm{bcd}$ & $97,81 \pm 0,58 \mathrm{a}$ & $99,19 \pm 0,36 \mathrm{ab}$ & 97,80 \\
\hline iprodiona & $96,94 \pm 0,53 a b c$ & $97,58 \pm 0,59 \mathrm{a}$ & $99,71 \pm 0,51 \mathrm{ab}$ & 98,35 \\
\hline dimetormorf & $91,76 \pm 1,33 \mathrm{~d}$ & $97,30 \pm 1,07 \mathrm{a}$ & $97,77 \pm 2,38 \mathrm{bc}$ & 95,97 \\
\hline tebufenozide & $97,01 \pm 1,06 \mathrm{abc}$ & $99,00 \pm 0,37 \mathrm{a}$ & $97,50 \pm 1,13 b c$ & 98,01 \\
\hline lambdacialotrina & $97,49 \pm 0,67 \mathrm{ab}$ & $98,73 \pm 1,03 \mathrm{a}$ & $98,14 \pm 0,27 \mathrm{bc}$ & 98,16 \\
\hline pirimicarb & $96,23 \pm 0,73 \mathrm{abcd}$ & $98,21 \pm 0,54 \mathrm{a}$ & $99,86 \pm 0,16 \mathrm{a}$ & 98,51 \\
\hline C.V. (\%) & 7,45 & 7,53 & 7,25 & \\
\hline
\end{tabular}

Médias seguidas da mesma letra, na coluna não diferem significativamente entre si pelo teste de Tukey a $5 \%$ de probabilidade.

EP = erro-padrão da média.

Ciênc. agrotec., Lavras. V.27, n.2, p.261-270, mar./abr., 2003 
TABELA 6 - Porcentagem média de emergência $( \pm \mathrm{EP})$ de Trichogramma pretiosum $\left(\mathrm{F}_{2}\right)$, da $\mathrm{L}_{10}$, provenientes de $\mathrm{F}_{1}$ tratadas nos estágios de ovo-larva, pré-pupa e pupa em ovos de Anagasta kuehniella.

\begin{tabular}{|c|c|c|c|c|}
\hline \multirow{3}{*}{$\begin{array}{c}\text { Produto } \\
\text { fitossanitário }\end{array}$} & \multicolumn{3}{|c|}{ Período de desenvolvimento do parasitóide } & \multirow{3}{*}{ Média } \\
\hline & $\begin{array}{c}\text { 0-24 h } \\
\text { (ovo-larva) }\end{array}$ & $\begin{array}{c}\text { 72-96 h } \\
\text { (pré-pupa) }\end{array}$ & $\begin{array}{c}\text { 168-192 h } \\
\text { (pupa) }\end{array}$ & \\
\hline & \multicolumn{3}{|c|}{ Porcentagem de emergência } & \\
\hline testemunha & $93,28 \pm 1,02 \mathrm{ab}$ & $97,72 \pm 0,86 \mathrm{a}$ & $93,48 \pm 0,73 \mathrm{a}$ & 95,07 \\
\hline clorfluazuron & $90,44 \pm 0,86 \mathrm{ab}$ & $95,08 \pm 0,36 \mathrm{a}$ & $91,67 \pm 0,43 \mathrm{a}$ & 92,53 \\
\hline teflubenzuron & $91,20 \pm 1,02 \mathrm{ab}$ & $95,43 \pm 0,87 \mathrm{a}$ & $92,11 \pm 0,40 \mathrm{a}$ & 93,03 \\
\hline triflumuron & $90,20 \pm 0,96 \mathrm{ab}$ & $96,15 \pm 2,21 \mathrm{a}$ & $92,87 \pm 0,76 \mathrm{a}$ & 93,29 \\
\hline B. thuringiensis & $92,08 \pm 1,25 \mathrm{ab}$ & $98,31 \pm 0,67 \mathrm{a}$ & $90,62 \pm 2,42 \mathrm{a}$ & 94,24 \\
\hline ciromazina & $90,97 \pm 1,28 \mathrm{ab}$ & $96,10 \pm 0,53 \mathrm{a}$ & $92,59 \pm 0,58 \mathrm{a}$ & 93,39 \\
\hline deltametrina & $94,21 \pm 4,65 \mathrm{a}$ & $96,48 \pm 1,33 \mathrm{a}$ & $87,94 \pm 0,59 a b$ & 93,28 \\
\hline acefato & $93,04 \pm 1,40 \mathrm{ab}$ & $96,90 \pm 1,06 \mathrm{a}$ & $88,57 \pm 1,07 \mathrm{ab}$ & 93,25 \\
\hline metamidofós & $86,92 \pm 1,05 b$ & $98,30 \pm 0,67 \mathrm{a}$ & $94,04 \pm 0,37 \mathrm{a}$ & 93,92 \\
\hline C.V. $(\%)$ & 9,71 & 8,81 & 9,22 & \\
\hline testemunha & $97,66 \pm 0,85 \mathrm{a}$ & $99,25 \pm 0,33 \mathrm{abc}$ & $96,67 \pm 0,55 \mathrm{a}$ & 98,02 \\
\hline Cartap & $96,91 \pm 1,04 \mathrm{a}$ & $96,96 \pm 0,65 \mathrm{bc}$ & $97,56 \pm 0,63 \mathrm{a}$ & 97,15 \\
\hline Benomil & $98,31 \pm 2,52 \mathrm{a}$ & $99,95 \pm 0,15 \mathrm{a}$ & $97,74 \pm 0,46 \mathrm{a}$ & 98,99 \\
\hline clorotalonil & $96,64 \pm 0,99 a$ & $99,93 \pm 0,26 \mathrm{a}$ & $96,60 \pm 1,18 \mathrm{a}$ & 98,27 \\
\hline mancozeb & $97,85 \pm 1,90 \mathrm{a}$ & $96,67 \pm 2,24 b c$ & $99,29 \pm 0,52 \mathrm{a}$ & 98,10 \\
\hline iprodiona & $98,68 \pm 0,35 \mathrm{a}$ & $99,65 \pm 0,47 \mathrm{ab}$ & $98,88 \pm 0,44 \mathrm{a}$ & 99,13 \\
\hline dimetormorf & $97,51 \pm 0,86 \mathrm{a}$ & $99,19 \pm 0,56 \mathrm{abc}$ & $98,03 \pm 0,35 \mathrm{a}$ & 98,33 \\
\hline tebufenozide & $90,56 \pm 5,56 b$ & $99,77 \pm 0,30 a b$ & $98,14 \pm 0,41 \mathrm{a}$ & 96,16 \\
\hline lambdacialotrina & $97,39 \pm 1,37 \mathrm{a}$ & $96,14 \pm 1,05 \mathrm{c}$ & $86,84 \pm 7,21 \mathrm{ab}$ & 93,46 \\
\hline pirimicarb & $94,66 \pm 4,33 \mathrm{a}$ & $98,74 \pm 1,33 \mathrm{abc}$ & $98,67 \pm 0,33 \mathrm{a}$ & 97,65 \\
\hline C.V. $(\%)$ & 10,40 & 9,22 & 11,41 & \\
\hline
\end{tabular}

Médias seguidas da mesma letra, na coluna não diferem significativamente entre si pelo teste de Tukey a $5 \%$ de probabilidade.

EP = erro-padrão da média. 


\section{CONCLUSÕES}

a) Os inseticidas triflumuron, clorfluazuron, deltametrina, Bacillus thuringiensis, lambdacialotrina, teflubenzuron, acefato, pirimicarb e ciromazina, e os fungicidas benomil, iprodiona, clorotalonil e dimetomorf, independente da linhagem, não reduziram a longevidade das fêmeas de $T$. pretiosum da geração $\mathrm{F}_{1}$.

b) Os inseticidas triflumuron, clorfluazuron, deltametrina, Bacillus thuringiensis, teflubenzuron, acefato, pirimicarb e ciromazina, e os fungicidas benomil, iprodiona, clorotalonil e dimetomorf não afetaram a razão sexual de indivíduos da geração $\mathrm{F}_{1}$, e nem a emergência de parasitóides da $\mathrm{F}_{2}$, independente do estágio de desenvolvimento e da origem da população de $T$. pretiosum.

c) T. pretiosum de Venda Nova do Imigrante, ES $\left(\mathrm{L}_{10}\right)$, mostraram-se mais susceptíveis que os de Alegre, ES $\left(\mathrm{L}_{9}\right)$ aos efeitos dos compostos avaliados.

d) De modo geral, a fase de pupa de T. pretiosum, independente da população, apresentou maior tolerância aos produtos fitossanitários avaliados.

e) Populações de $T$. pretiosum responderam de forma diversa aos compostos avaliados e, dessa forma, como estratégia do MIP, recomenda-se a realização de novos testes para outras populações dessa espécie.

\section{REFERÊNCIAS BIBLIOGRÁFICAS}

AMAYA-NAVARRO, M. Trichogramma spp.: producción, uso y manejo en Colombia. Valle del Cauca: [s.n.], 1998. 176 p.

CAMPBELL, C. D.; WALGENBACH, J. F.; KENNEDY, G. G. Effect of parasitoids on lepidopterous pests in insecticide-treated and untreated tomatoes in western North Carolina. Journal of Economic Entomology, College Park, v. 84, n. 6, p. 1662-1667, 1991.

CARVALHO, G. A. de.; TIRONI, P.; RIGITANO, R. L. O.; SALGADO, L. O. Seletividade de inseticidas reguladores de crescimento de insetos a Trichogramma pretiosum Riley (Hymenoptera: Trichogrammatidae). Anais da Sociedade Entomológica do Brasil, Jaboticabal, v. 23, n. 3, p. 431-434, 1994.

FARIA JÚNIOR, P. A. J. Controle biológico da traça do tomateiro pela "FRUTINOR". In: SIMPÓSIO DE
CONTROLE BIOLÓGICO, 3., 1992, Águas de Lindóia. Anais... Jaguariúna: EMBRAPA/CNPDA, 1992. p. 61-63.

HAJI, F. N. P. Histórico e situação atual da traça-dotomateiro nos perímetros irrigados do submédio São Francisco. In: SIMPÓSIO DE CONTROLE BIOLÓGICO, 3., 1992, Águas de Lindóia. Anais... Jaguariúna: EMBRAPA/CNPDA, 1992. p. 57-59.

HAJI, F. N. P. Controle biológico da traça do tomateiro, com uso de Trichogramma, na região do submédio São Francisco. In: SIMPÓSIO DE CONTROLE BIOLÓGICO, 5., 1996, Foz do Iguaçu. Anais... Londrina: EMBRAPA/CNPSA, 1996. p. 355-360.

HAJI, F. N. P. Controle biológico da traça do tomateiro com Trichogramma no Nordeste do Brasil. In: PARRA, J. R. P.; ZUCCHI, R. A. (Eds.). Trichogramma e o controle biológico aplicado. Piracicaba: FEALQ, 1997. cap. 12, p. 319-324.

LANGE, W. H.; BRONSON, L. Insect pests of tomatoes. Annual Review of Entomology, Stanford, v. 26, p. 345-371, 1981.

PARRA, J. R. P. Seletividade de alguns produtos químicos utilizados para o controle de Scrobipalpuloides absoluta (Meyrick) ao parasitóide Trichogramma pretiosum Riley: relatório de pesquisa. Piracicaba: FEALQ, 1994. 39 p.

WETZEL, C.; DICKLER, E. Side effects of sulphur and a natural pyrethroid on Trichogramma dendrolimi Matsumura (Hym., Trichogrammatidae) in apple orchards. In: INTERNATIONAL ORGANIZATION FOR BIOLOGICAL CONTROL OF NOXIOUS ANIMALS AND PLANTS. Working group "pesticides and beneficial organisms. Montfavet, 1994. p. 123-131. (Bulletin SROP, 17/10).

ZAKI, F. N.; GESRAHA, M. A. Evaluation of zertel and diflubenzuron on biological aspects of the egg parasitoid, Trichogramma evanescens Westw. and the aphid lion Chrysoperla carnea Steph. Journal of Applied Entomology, Hamburg, v. 104, p. 63-69, 1987. 